\title{
Influence of surface oxidation on the valence electron energy-loss spectrum of wurtzite aluminum nitride
}

\author{
Michael R. S. Huang, ${ }^{1}$ Rolf Erni, ${ }^{2}$ and Chuan-Pu Liu ${ }^{1,3, a)}$ \\ ${ }^{1}$ Department of Materials Science and Engineering, National Cheng-Kung University, Tainan 701, Taiwan \\ ${ }^{2}$ Electron Microscopy Center, Empa, Swiss Federal Laboratories for Materials Science and Technology, \\ 8600 Dübendorf, Switzerland \\ ${ }^{3}$ Center for Micro/Nano Science and Technology, National Cheng Kung University, Tainan 701, Taiwan; \\ Research Center for Energy Technology and Strategy, National Cheng Kung University, Tainan 701, Taiwan; \\ and Advanced Optoelectronic Technology Center, National Cheng Kung University, Tainan 701, Taiwan
}

(Received 29 December 2012; accepted 22 January 2013; published online 11 February 2013)

\begin{abstract}
The influence of surface oxidation on the low-loss spectrum of aluminum nitride (AIN) is investigated in electron energy-loss spectroscopy with scanning transmission electron microscopy. Contrary to intrinsic bulk AlN, oxidized AlN exhibits considerable spectral broadening both in the full width at half maximum of bulk plasmon and the subsidiary features. The modification in the low-loss lineshapes due to oxidation significantly complicates the determination of the dielectric function intrinsic to AlN. Simulations based on dielectric theory qualitatively consist with the experimental results while incorporating thick overlayers, further suggesting that the surface oxide of AlN can be rough and porous in nature. (C) 2013 American Institute of Physics.

[http://dx.doi.org/10.1063/1.4790395]
\end{abstract}

Wurtzite aluminum nitride (AIN), with a wide direct bandgap up to $6.2 \mathrm{eV}$, has recently attracted considerable attention, because of its promising optoelectronic applications, especially in energy related fields such as highbrightness light-emitting diodes and full spectrum solar cells when alloyed with other III-V nitride semiconductors. ${ }^{1}$ To further enhance the functionality of this material, comprehensive understanding of the fundamental electronic structures is required. Access to the electronic structures can be achieved by examining the corresponding dielectric function (DF), which essentially determines the response to external electromagnetic perturbations such as light or electrons. Compared to conventional optical methods, electron energyloss spectroscopy (EELS) with scanning transmission electron microscopy (STEM) can alternatively provide spectral information over a significantly wider frequency range accompanied by excellent spatial resolution, which substantially facilitates the exploration for nanomaterials. ${ }^{2,3}$ Therefore, extensive studies on AlN dielectric properties have been made accordingly. ${ }^{4,5}$ However, the majority of earlier works in the literature do not appear to represent the intrinsic properties of bulk AIN accurately, since the influence of surface oxidation was not adequately verified.

Owing to the strong chemical affinity of AlN for oxygen, AlN is easily prone to oxidation, forming an amorphous $\mathrm{Al}_{2} \mathrm{O}_{3}$ $\left(\mathrm{a}-\mathrm{Al}_{2} \mathrm{O}_{3}\right)$ surface layer by reactions with moisture or oxygen even at room temperature, ${ }^{6-8}$ despite the superior stability that is generally regarded. The current results are thus argued to be more consistent with the oxidized material. ${ }^{7-9}$ The available examinations into oxidation with EELS were concentrated, however, primarily on the variation of the core-loss features such as $\mathrm{Al}-\mathrm{L}_{2,3}$ and N-K edges. ${ }^{6,8}$ None were dedicated to the low-loss regime, which is essential for subsequent dielectric property investigation. Furthermore, certain electronic excita-

\footnotetext{
${ }^{\text {a) }}$ Author to whom correspondence should be addressed. Electronic mail: cpliu@mail.ncku.edu.tw.
}

tions, designated to originate from $\mathrm{Al}$ vacancies or localized states, ${ }^{9,10}$ were commonly observed below the fundamental bandgap, further revealing their defective crystallinity. In addition, concerning the calculations of the DF of AlN using EELS, the relativistic effects such as retardation and Čerenkov radiation ( $\breve{\mathrm{C}})$, which can potentially induce spectral artifacts near the bandgap especially for semiconductors, ${ }^{11,12}$ were largely ignored. ${ }^{4,5}$ Thus previous derivations of DFs from the low-loss spectrum remain unreliable and must be reexamined.

In this study, the low-loss spectrum of bulk AlN is clearly demonstrated to be significantly modified by oxygen incorporation, which subsequently complicates the accurate determination of the DF through the Kramers-Kronig analysis (KKA). With careful consideration for both surface oxidation and retardation effects, the corresponding complex DF, closely representative of the intrinsic and oxygen-free bulk AlN, is proposed. Theoretical simulations have also been made in support of the experimental observations.

Commercial AlN nanoparticles (NPs) with well established chemical compositions and a crystallographic structure (wurtzite) were employed in this study. For obtaining the low-loss spectrum representative of intrinsic bulk AlN, considerable attention has been paid to minimizing the surface oxidation. Samples were prepared by dispersing AIN powders directly on $\mathrm{Cu}$ grids with a lacy carbon network without any aqueous process, ${ }^{7}$ followed by plasma cleaning to remove carbon contamination, and were then loaded into TEM with minimal exposure to the atmosphere. Spectroscopic analysis was performed in STEM mode with a probe size of approximately $0.1-0.12 \mathrm{~nm}$, in a probe-Cs-corrected TEM JEOL $2100 \mathrm{~F}$ system operated at $200 \mathrm{kV}$, equipped with a Gatan ENFINA spectrometer. Throughout the STEMEELS acquisition, the energy resolution was optimized to be $0.6 \mathrm{eV}$ along with respective convergence and collection semi-angles of 13 and 7.4 mrad.

Fig. 1(a) shows the spectrum acquired from a nearly oxygen-free region, where no oxygen K-edge signal can be 

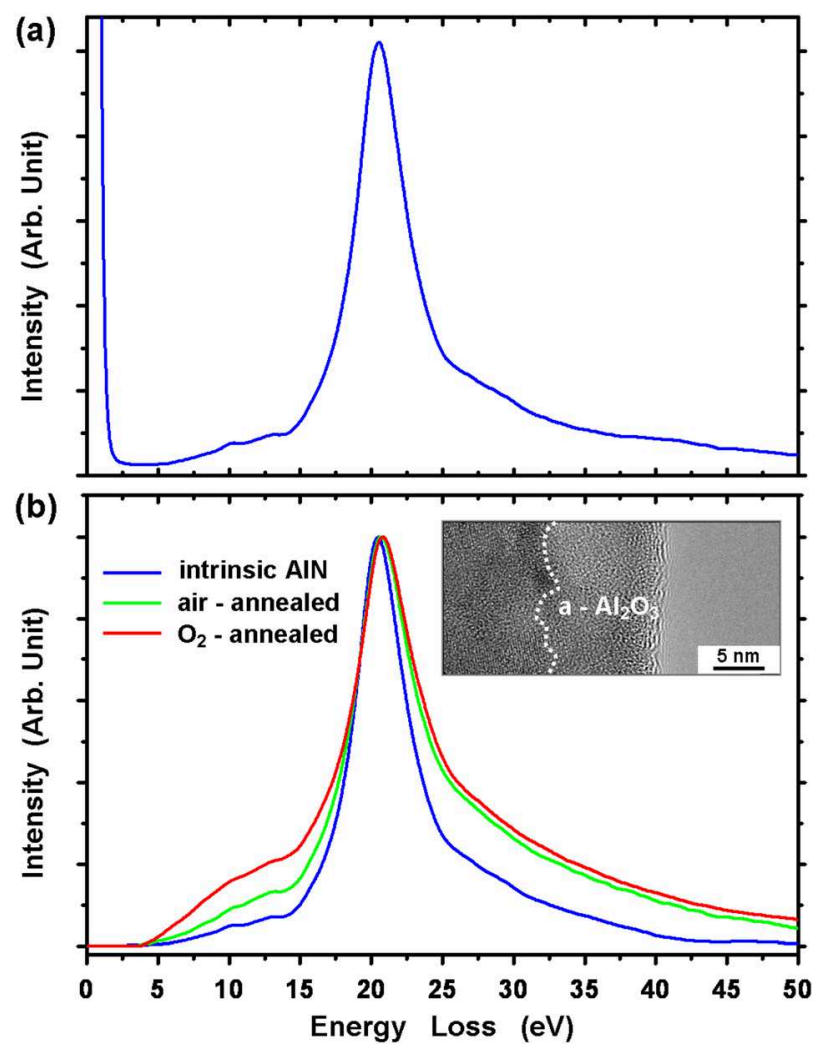

FIG. 1. (a) Low-loss spectrum of AlN acquired from a nearly oxygen free region. (b) Comparison of the spectra from intrinsic AlN (blue), atmosphere (green), and $\mathrm{O}_{2}$ (red) annealed samples, with zero-loss peaks and plural scattering deconvoluted. The inset shows a TEM bright-field image of a- $\mathrm{Al}_{2} \mathrm{O}_{3}$ overlayers at the edge of an oxidized NP.

detected, indicating rather low oxygen content below the EELS detection limit, typically within few atomic percent. It consists of a dominant bulk plasmon (BP) peak located at $20.4 \mathrm{eV}$ with a narrow full width at half maximum (FWHM) of about $4.4 \mathrm{eV}$, along with some subsidiary interband transitions at $10,13,15-16,26-28$, and $37-44 \mathrm{eV}$. Specifically, no features are found below the fundamental bandgap, where no excitations should be expected for perfect crystallinity. This low-loss spectrum thereby is believed to represent the intrinsic AlN bulk property. To further illustrate the influence of oxidation on the spectral features, the low-loss spectrum of intrinsic AlN (Fig. 1(a)) is then compared to the results obtained from purposely oxidized samples, annealed at $200{ }^{\circ} \mathrm{C}$ in the atmosphere (green line) and in $\mathrm{O}_{2}$ (red line) for $2 \mathrm{~h}$, respectively (Fig. 1(b)). More serious oxidation was confirmed in the $\mathrm{O}_{2}$-annealed sample than in the air-annealed one through measuring the intensity of the oxygen K-edge. The surface oxide remains amorphous according to the TEM bright-field image and electron diffraction (inset in Fig. 1(b)). For clarity, these spectra were collected from regions of similar thicknesses and deconvoluted with both zero-loss peaks and plural scattering removed, accompanied by normalization to the intensity maxima.

Fig. 1(b) reveals that with increasing oxidation the spectrum is characterized by a gradual increase in the FWHM of the $\mathrm{BP}$, from about $4.4 \mathrm{eV}$ for the intrinsic case (blue) to over $6.4 \mathrm{eV}$ for the oxidized AlN (red), together with prominent broadening on both tails of the plasmon peak. The BP maximum additionally shows a systematic shift toward the higher energy side with oxidation. Actually, a majority of the as-received NPs exhibit similar spectral features as the oxidized cases with only few resembling intrinsic AlN as shown in Fig. 1(a), which experimentally supports the high oxidation affinity of AlN even handled with caution at room temperature. Note that most of the earlier EELS works on AlN coincides with the results of the oxidized AlN obtained here (green and red lines in Fig. 1(b)), especially in the spectral width, which strongly suggests the possible oxidation of these samples. ${ }^{4,5}$

For understanding the inherent physics of the low-loss spectra, the energy-dependent complex DF $\left(\varepsilon=\varepsilon_{1}+\mathrm{i} \varepsilon_{2}\right)$ is thus derived. To accurately extract the dielectric information, the KKA has been performed accordingly with careful consideration for relativistic contributions by evaluating sample thicknesses. The solid lines in Fig. 2(a) show the calculated DF of intrinsic AlN according to the blue curve in Fig. 1(b). Compared with the reported optical data ${ }^{7,9}$ determined from a single-crystalline AIN film with oxygen content controlled and mitigated (Fig. 2(b)), qualitative consistence can be achieved either in the profile shape or the relative intensity between $\varepsilon_{1}$ and $\varepsilon_{2}$. Distinctively the absence of an additional absorption hump in $\varepsilon_{2}$ below the bandgap further verifies the reliability of the extracted DF. The remaining difference in the DF structure might result from both the limited energy resolution in the EELS and the angular-integrated signals under the converged illumination STEM mode, which can cause some momentum-dependent or anisotropy-related features in the spectra as well as in the DF to be smeared.

With surface oxidation incorporated, the corresponding DF (dashed lines in Fig. 2(a)), however, exhibits a considerable modification in the lineshape together with an appreciable

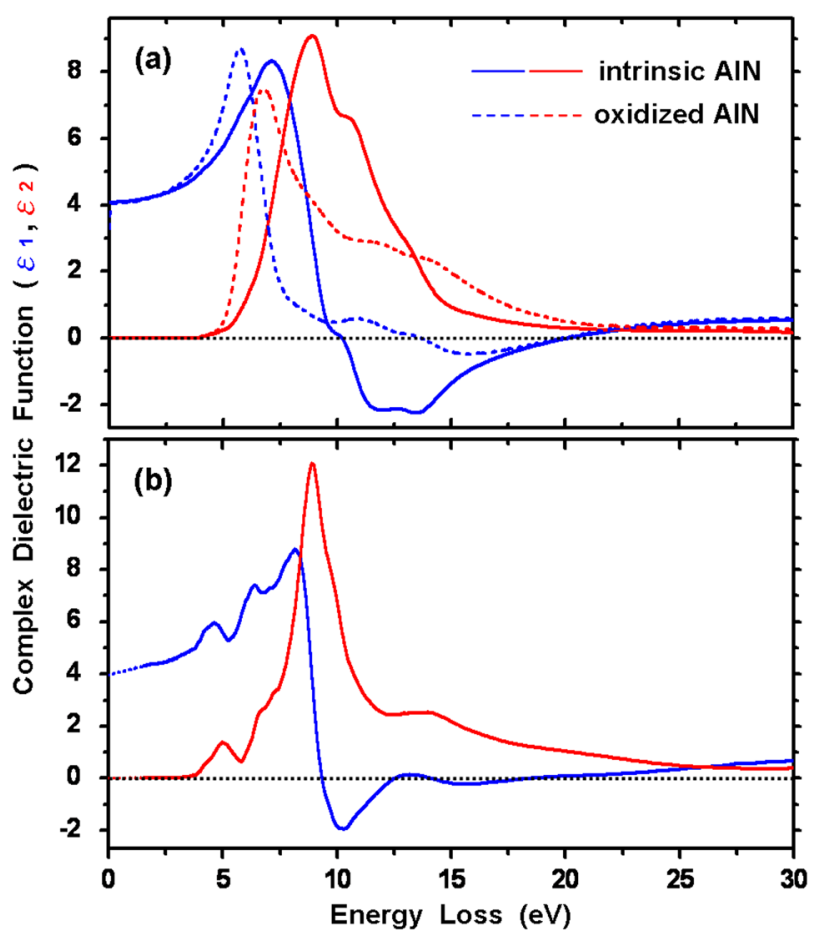

FIG. 2. (a) Experimentally derived complex DFs $\left(\varepsilon=\varepsilon_{1}+\mathrm{i} \varepsilon_{2}\right)$ of intrinsic (solid line) and oxidized (dashed line) AlN. (b) Computed DF according to the optically determined refractive index and extinction coefficient in Refs. 7 and 9 . 
shift toward the lower energy side, particularly below $20 \mathrm{eV}$. Meanwhile, the maximum magnitudes of the real and imaginary parts of the DF are reversed $\left(\varepsilon_{1 \text { max. }}>\varepsilon_{2 \text { max. }}\right)$, in stark contrast to the results of both the intrinsic AlN (solid lines in Fig. 2(a)) and the optical reference (Fig. 2(b)). Moreover, examination of another well-recognized EELS work shows that the behavior of the proposed $\mathrm{DF}^{5}$ is more analogous to the oxidized case, rather than to intrinsic AlN. Thus a more profound influence of surface oxidation on the DF determination is clearly demonstrated.

To further assist the interpretation of the observed spectral variation with oxidation, simulations with dielectric theory have been conducted. The employed Bolton-Chen equations, describing the energy-loss probability of fast electrons transmitting a thin film, include bulk dielectric loss and contributions from surface, interface, and retardation as well. ${ }^{12-14}$ This calculation can also be applied to multilayer systems and, hence, is suitable for the present study. Here the modeled material structure consists of a $60-\mathrm{nm}$ AlN slab, which can intrinsically reflect bulk characters and approximate the experimental magnitude, with the oxide overlayer of various thicknesses on each side. The input DF for AlN is experimentally derived, while that for the $\mathrm{a}-\mathrm{Al}_{2} \mathrm{O}_{3}$ is adopted from the literature. ${ }^{15}$

Fig. 3(a) summarizes the calculated results with intensity normalized, in which the gray-shaded region represents the intrinsic AlN profile, whereas the influence of surface oxidation is illustrated by solid lines. With increasing oxide thickness, systematic broadening can be readily identified in both the BP and peripheral features, which satisfactorily agrees

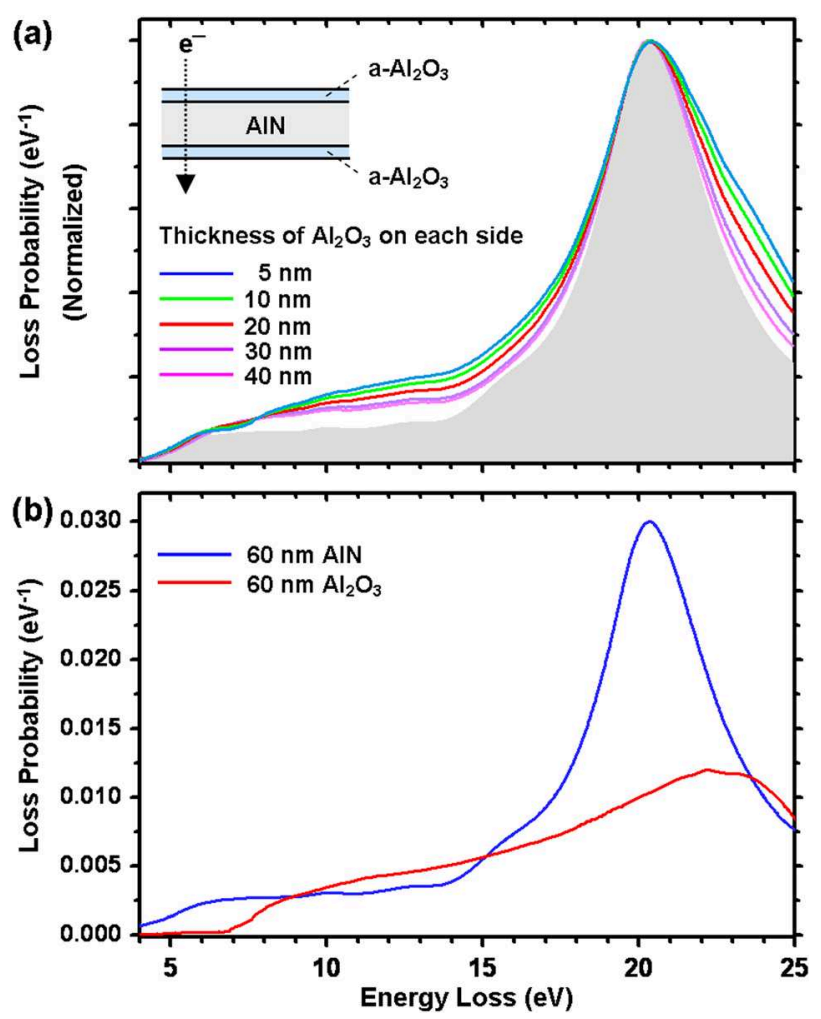

FIG. 3. (a) Simulated low-loss spectra of a $60-\mathrm{nm}$ bulk AIN slab with various thicknesses of a- $\mathrm{Al}_{2} \mathrm{O}_{3}$ overlayer. The schematic representation of the multilayer model utilized is also shown in the inset. (b) Calculated lossprobabilities of the respective bulk $\mathrm{AlN}$ and $\mathrm{a}-\mathrm{Al}_{2} \mathrm{O}_{3}$ of the same $60 \mathrm{~nm}$. with the experimental observations. It thus computationally proves that the surface oxidation can indeed cause the broadening of intrinsic AlN. Another noticeable point is that rather thick overlayers ( $>5 \mathrm{~nm}$ on each side) are required to cause such a change. In addition, despite the higher BP energy of a- $\mathrm{Al}_{2} \mathrm{O}_{3}$ at $23 \mathrm{eV}$, the predicted $\mathrm{BP}$ reveals merely a small blue-shift $(<0.3 \mathrm{eV})$ even with much thicker oxide layers almost reaching $60 \%$ of the total thickness.

Further comparing the spectra of $\mathrm{AlN}$ and $\mathrm{a}-\mathrm{Al}_{2} \mathrm{O}_{3}$ of the same $60 \mathrm{~nm}$ calculated separately (Fig. 3(b)), it is found that the AlN substantially dominates over the a- $\mathrm{Al}_{2} \mathrm{O}_{3}$, especially near the energy regime where its BP falls. This is also well revealed in the intensity magnitude, in which the AlN peaks at $0.03 \mathrm{eV}^{-1}$ whereas the maximum of a- $\mathrm{Al}_{2} \mathrm{O}_{3}$ is only $0.01 \mathrm{eV}^{-1}$, implying that the AlN plasmon has higher excitation probability and thus can be naturally more pronounced. It is then clear that, because of the relatively weak excitations of a- $\mathrm{Al}_{2} \mathrm{O}_{3}$, the resultant low-loss spectra exhibit mainly broadened features along with a marginal shift of the corresponding BP instead of showing drastic interference in their lineshapes, even with a large amount of oxide incorporated. Another possible explanation for the highly estimated overlayer thickness can be associated with either nonstoichiometry or incomplete oxidation. The formed surface oxide can be composed of a- $\mathrm{Al}_{2} \mathrm{O}_{3}$ with partial $\mathrm{AlN}$, forming Al-oxynitride, and the actual loss-probability profile may lie between the pure $\mathrm{a}-\mathrm{Al}_{2} \mathrm{O}_{3}$ and AlN (Fig. 3(b)), which reduces the computational thickness necessary to conform to the experimental results.

Therefore, based upon the above analysis, it is concluded that the surface of AlN may not be as stable as intuitively recognized. Unlike the dense and self-limited $\mathrm{SiO}_{2}$ grown on $\mathrm{Si}$, typically $<2 \mathrm{~nm},{ }^{16}$ the property of natural aluminum oxide can be rough and porous, which significantly facilitates continuous oxidation. The earlier proposed low-loss spectra of $\mathrm{AlN}^{4,5}$ characterized by broad lineshapes with relatively large BP FWHM ( $>6 \mathrm{eV})$, could incorporate quite thick oxide layers on the surface already and thus do not represent the intrinsic characteristics of AlN. It should be mentioned that the simulations utilized in this study, with a simplified model considering the oxide overlayers on both sides, actually exclude the main oxygen impurities dissolved in AlN bulk lattices, especially at high temperature processes. ${ }^{6,17}$ Such compositional fluctuations, although uninvolved in the simulations here, are believed to have impacts on the low-loss features principally through the alteration of valence electron density ${ }^{18}$ and may account for the large discrepancy in the measured BP energy, ranging from 20 to over $21 \mathrm{eV}$.,5,10,19 Moreover, some induced structural defects, such as stacking faults, ${ }^{20}$ can also damp the BP resonance and decrease the relaxation time, further leading to the increase in the $\mathrm{BP}$ width as well. These factors, however, tangle interactively and are difficult to be experimentally differentiated.

In summary, the correlation between the variation in the low-loss features of bulk AlN and surface oxidation has been established using STEM-EELS. With the incorporation of oxide overlayers, a considerable spectral broadening both in the BP and in the subsidiary characteristics can be identified, which further complicates the DF determination of intrinsic AlN. The experimental observations in the low-loss regime 
can be well reproduced in theoretical simulations, which also imply the porous and rough properties of natural oxide formed on the AlN surface.

This research is financially supported from the National Science Council, Taiwan, under Grant No. NSC-98-2221E-006-079-MY3. The authors would also acknowledge the Center for Micro/Nano Science and Technology in National Cheng Kung University, and the NSC Core Facilities Laboratory for Nano-Science and Nano-Technology in KaohsiungPingtung for the equipment and technical support.

${ }^{1}$ Y. Taniyasu, M. Kasu, and T. Makimoto, Nature (London) 441(7091), 325 (2006).

${ }^{2}$ M. R. S. Huang, R. Erni, H. Y. Lin, R. C. Wang, and C. P. Liu, Phys. Rev. B 84(15), 155203 (2011).

${ }^{3}$ R. Erni and N. D. Browning, Ultramicroscopy 104(3-4), 176 (2005).

${ }^{4}$ A. D. Dorneich, R. H. French, H. Mullejans, S. Loughin, and M. Ruhle, J. Microsc. 191, 286 (1998)

${ }^{5}$ G. Brockt and H. Lakner, Micron 31(4), 435 (2000).
${ }^{6}$ M. Sternitzke, J. Am. Ceram. Soc. 76(9), 2289 (1993).

${ }^{7}$ E. D. Palik, Handbook of Optical Constants of Solids (Academic, New York, 1985).

${ }^{8}$ M. MacKenzie and A. J. Craven, J. Phys. D 33(14), 1647 (2000).

${ }^{9}$ D. J. Jones, R. H. French, H. Mullejans, S. Loughin, A. D. Dorneich, and P. F. Carcia, J. Mater. Res. 14(11), 4337 (1999).

${ }^{10}$ M. Gautier, J. P. Duraud, and C. Legressus, Surf. Sci. 178(1-3), 201 (1986).

${ }^{11}$ M. Stoger-Pollach, H. Franco, P. Schattschneider, S. Lazar, B. Schaffer, W. Grogger, and H. W. Zandbergen, Micron 37(5), 396 (2006).

${ }^{12}$ R. Erni, S. Lazar, and N. D. Browning, Ultramicroscopy 108(3), 270 (2008).

${ }^{13}$ E. Kroger, Z. Phys. 216(2), 115 (1968).

${ }^{14}$ J. P. R. Bolton and M. Chen, Ultramicroscopy 60(2), 247 (1995).

${ }^{15}$ C. H. Chen and J. Silcox, Solid State Commun. 17(3), 273 (1975).

${ }^{16}$ C. H. Chen, J. Silcox, and R. Vincent, Phys. Rev. B 12(1), 64 (1975).

${ }^{17}$ H. Buhr, G. Muller, H. Wiggers, F. Aldinger, P. Foley, and A. Roosen, J. Am. Ceram. Soc. 74(4), 718 (1991).

${ }^{18}$ J. Park, S. Heo, J. G. Chung, H. Kim, H. Lee, K. Kim, and G. S. Park, Ultramicroscopy 109(9), 1183 (2009).

${ }^{19}$ C. G. Olson, J. H. Sexton, D. W. Lynch, A. J. Bevolo, H. R. Shanks, B. N. Harmon, W. Y. Ching, and D. M. Wieliczka, Solid State Commun. 56(1), 35 (1985).

${ }^{20}$ D. Dorignac, A. Mazel, Y. Kihn, J. Sevely, B. Aspar, B. Armas, and C. Combescure, J. Eur. Ceram. Soc. 13(4), 345 (1994). 